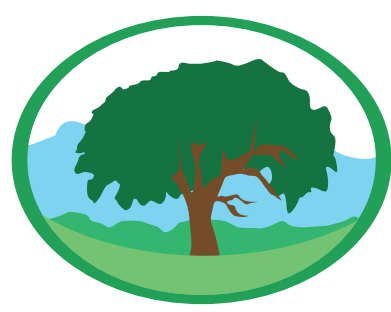

\title{
TRATAMENTO DE EFLUENTE CONTENDO FLUFENOXURON GERADO PELA LAVAGEM E DESCARTE DAS APLICAC̣ÕES AÉREAS
}

\author{
FURTADO, R. D. ${ }^{1} ;$ HOFF, RODRIGO B. ${ }^{1}$ \\ 'Ministério da Agricultura, Pecuária e Abastecimento.
}

Palavras-chave: ozônio, oxidação, pátio de descontaminação.

\section{Resumo}

A legislação ambiental brasileira, subsidiada pela Instrução Normativa n0 02/2008 do Ministério da Agricultura, Pecuária e Abastecimento, obriga que as empresas operadoras aeroagrícolas realizem o tratamento dos efluentes gerados após a lavagem das aeronaves. Para este fim, é sugerido pela legislação o uso de um sistema de degradação baseado na ozonização. Avaliou-se a eficiência do sistema recomendado pela legislação em promover a degradação do princípio ativo flufenoxuron (inseticida Cascade ${ }^{\circledR} 100$ ), onde foram analisadas a concentração e a quantidade em massa da molécula em todas as etapas do sistema. Foram avaliados dois tratamentos distintos, usando 1,0 e 2,0 g de ozônio/hora, em um sistema industrial de geração de ozônio, usando três períodos de ozonização diferentes (1, 3 e 6 horas), em temperatura ambiente e pH em torno de 6,0. Constatou-se que, em cada lavagem, é gerado um volume médio de $132 \mathrm{~L}$ de efluente $(112 \mathrm{~L}$ de água mais $20 \mathrm{~L}$ de calda residual). Após sofrer os tratamentos com 1,0 e 2,0 g de $\mathrm{O}_{3}$ /hora foram obtidos, respectivamente, os seguintes resultados: diminuição da concentração de flufenoxuron no efluente $(-82,8$ e -86,0\%) e decréscimo na massa de flufenoxuron $(-44,8$ e $-63,2 \%)$. 0 sistema mostrou-se eficiente no tratamento do efluente contendo resíduos do inseticida Cascade ${ }^{\circledR} 100$.

\section{TREATMENT OF EFFLUENT CONTAINING FLUFENOXURON GENERATED BY WASHING AND DISPOSAL OF AERIAL APPLICATIONS}

Keywords: ozone, oxidation, airstrip decontamination

\begin{abstract}
The Brazilian environmental legislation, subsidized by Normative Instruction No. 02/2008 of the Ministry of Agriculture, Livestock and Food Supply, obliges the aeroagriculture operators to treat the effluents generated after washing the aircraft. To this end, the use of a degradation system based on ozonation is suggested by legislation. The efficiency of the system recommended by the legislation in promoting the degradation of the active principle flufenoxuron (Cascade ${ }^{\circledR} 100$ insecticidel was evaluated through the analysis of pesticide concentration and the mass quantity in all the system stages. Two different treatments were evaluated using 1.0 and $2.0 \mathrm{~g}$ ozone/hour. It was found that an average volume of $132 \mathrm{~L}$ of effluent (112 $\mathrm{L}$ of water plus $20 \mathrm{~L}$ of residual pesticide formulation) is generated for each wash. After treatment with 1.0 and $2.0 \mathrm{~g} \mathrm{O}_{3}$ /hour respectively, the following results were obtained: reduction of the flufenoxuron concentration in the effluent (-82.8 and $-86.0 \%$ ); decrease on the amount of flufenoxuron (-44.8 and $-63.2 \%)$. The system proved to be efficient in the treatment of effluent containing flufenoxuron residues.
\end{abstract}




\section{INTRODUÇÃO}

A aplicaçáo de agrotóxicos através de aeronaves agrícolas é realizada, geralmente, ou em culturas de grande escala, e/ou pela impossibilidade de trânsito de pulverizadores terrestres sobre o solo (ANTUNIASSI, 2015). A cultura da soja é uma das que mais demanda este serviço no estado do Rio Grande do Sul (BUENO, M. R.; CUNHA; SANTANA, DE, 2017).

O flufenoxurom é um inseticida da classe das benzoiluréias e que é usado na pulverizaçáo aérea de lavouras de soja para o combate à Lagarta-da-soja (Anticarsia gemmatalis) (CARMO; BUENO, A. F; BUENO, R. C. O. F., 2010). Uma das formulaçōes de flufenoxurom comumente utilizada para este fim é o produto Cascade 100.

O flufenoxurom é um agrotóxico de alta toxicidade para humanos (JEONG et al., 2014; WOO; LIM, 2015) e tem sido implicado também na toxicidade sobre as abelhas (COSTA et al., 2014). Foram publicados poucos trabalhos a respeito de sua degradaçáo no ambiente e quais produtos de transformação são gerados (KAMEL et al., 2007; MAILLARD et al., 2011). O flufenoxurom tem sido detectado em inúmeras amostras de vegetais e em águas subterrâneas, demonstrado a importância dos processos de tratamento e degradaçáo deste composto (BARRANCO et al., 2007; GIL et al., 2006).

Os efluentes gerados pela lavagem das aeronaves e de seus equipamentos de aplicação de agrotóxicos necessitam ser tratados antes de serem lançados ao meio ambiente, para evitar que se tornem fonte pontual de contaminação. Para evitar o risco desse impacto ambiental, são necessárias medidas especiais de tratamento. $\mathrm{O}$ lançamento de efluente náo tratado diretamente no solo provoca contaminação pontual na área de operaçáo aeroagrícola e, devido à alta concentração de agrotóxicos neste resíduo, todo o entorno da área da lavagem das aeronaves apresentará contaminaçāo (MINISTÉRIO DA AGRICULTURA, PECUÁRIA E ABASTECIMENTO, 2008).

Os agrotóxicos podem ser naturalmente degradados, através da oxidação gerada pelo oxigênio livre da atmosfera. Logo, qualquer método artificial que potencialize injeção de oxigênio livre em um meio com alta concentraçáo de agrotóxicos irá promover o aceleramento da degradaçáo destes compostos (OLLER; MALATO; SÁNCHEZ-PÉREZ, 2011).

Tecnologias de oxidação química têm sido preconizadas para oxidar efluentes de estaçóes de lavagem, uma vez que podem ser instalaçóes portáteis, gerando o ozônio in situ, com alta eficiência oxidativa e baixo tempo de retenção (STATHIS et al., 2011).

O efluente produzido pelo descarte dos restos da calda não pulverizada e pela lavagem das aeronaves possui três características básicas que são: a) baixo volume de líquidos, b) alta concentraçáo de princípios ativos de agrotóxicos proibidos de serem lançados diretamente no meio ambiente e, c) grandes distâncias de centros recebedores deste tipo de efluente, fazendo do tratamento no próprio local uma das únicas opçóes econômica e operacionalmente viáveis (FURTADO, 2012; RICE et al., 2005).

O objetivo deste trabalho foi estudar a geração de efluentes a partir da aplicação aérea do inseticida Cascade $^{\circ} 100$ e analisar a eficiência do sistema-piloto de tratamento com ozônio conforme proposto na Instrução Normativa no 02/2008 do MAPA. O efluente estudado foi produzido pela lavagem de aeronaves agrícolas e pelo descarte das sobras das aplicaçôes do inseticida Cascade ${ }^{\circ} 100$ e seu princípio ativo flufenoxurom.

\section{MATERIAL E MÉTODOS}

\section{-Efluentes}

Foi utilizada formulação comercial do inseticida Cascade 100 (BASF S.A., Resende, RJ, Brasil), contendo $10,0 \%(\mathrm{~m} / \mathrm{v})$ de flufenoxurom, comercializado na forma de concentrado emulsionável. O produto foi diluído para a produção de calda conforme recomendação do fabricante ("Inseticida Cascade 100 : BASF", [s.d.]).

A amostra de efluente foi obtida a partir da água utilizada para a lavagem externa e interna de aeronave tipo Ipanema 2002B. O volume de água utilizado na lavagem das aeronaves foi primeiramente estimado com base na vazão e tempo de operação da máquina lavadora. $\mathrm{O}$ mesmo foi confirmado experimentalmente pelo recolhimento de toda a água utilizada no processo em um tonel de capacidade de $200 \mathrm{~L}$. O volume foi 
posteriormente aferido usando frasco graduado de polipropileno de $5 \mathrm{~L}$. A quantidade média de água utilizada no processo de lavagem foi de 112,8 litros. A este volume somam-se cerca de 20 litros de agrotóxico, correspondente ao volume morto do tanque, abaixo do qual a bomba hidráulica não consegue mais captar, provocando sobra da calda no hopper da aeronave. O volume final de efluente possuiu, portanto, volume de 132,8 litros, o qual foi usado em sua totalidade como amostra para avaliação da eficácia do sistema de descontaminação.

As amostras foram coletadas junto a empresas de aviação agrícola situadas nas cidades de Rosário do Sul (RS) e Dom Pedrito (RS), durante a safra 2009/2010. Foram coletadas amostras de 400 lavagens, obtendo assim dados representativos das características operacionais das aplicaçóes aéreas realizadas no sul do Rio Grande do Sul.

\section{-Sistema de tratamento}

O sistema de tratamento utilizado neste trabalho tem como base o modelo de pátio de descontaminação previsto na legislação brasileira (MINISTÉRIO DA AGRICULTURA, PECUÁRIA E ABASTECIMENTO, 2008). Este sistema consiste em um piso de lavagem impermeabilizado e com contenção lateral, onde a aeronave passa pelo processo de lavagem com água após a operação. Toda a água utilizada neste piso é drenada por um cano e chega até um tanque de decantação igualmente impermeabilizado. Deste tanque, o efluente gerado na lavagem é bombeado para o reator de ozonização. Após o tempo de residência neste reator, o efluente é direcionado ao leito de volatilizaçáo, onde permanece até a evaporação total.

A Figura 1 mostra croqui ilustrativo do pátio de descontaminação utilizado no experimento, bem como as diferentes partes do sistema e os locais/momentos da retirada da cada amostra.

Figura 1. Croqui com o pátio de descontaminação e o local da amostragem durante o fluxo do processo. Amostra 0 - piso de lavagem; Amostra 1 - cano para drenagem; Amostra 2 - tanque de decantação; Amostra 3,4 e 5 - reator de ozonização; Amostra 6 - leito de volatilização

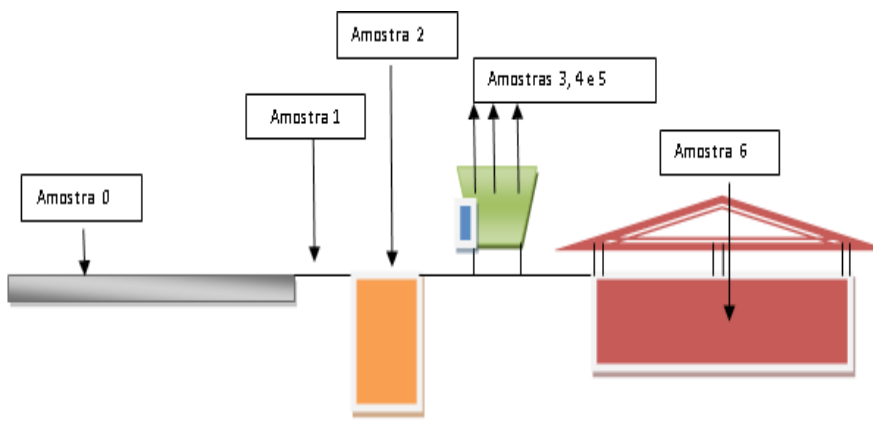

\section{-Descontaminação dos efluentes}

Realizou-se a passagem da amostra de efluentes gerados pela lavagem de aeronave agrícola após aplicação do produto Cascade 100 (contendo 10,0\% (m/v) de flufenoxurom) pelo sistema de tratamento, tendo sido coletadas amostras para análise da concentração e da carga do princípio ativo, com objetivo de se comparar o efeito de cada compartimento (tanque de decantação, reator de ozonizaçáa e leito de volatilização) do sistema de tratamento e da aplicação de diferentes concentraçóes de ozônio (1,0 e 2,0 g de $\mathrm{O}_{3}$ /hora) sobre a degradação do inseticida flufenoxurom. A vazão do ozônio foi mantida constante em 9,0 L/min para os dois níveis de concentração de ozônio avaliados. Para padronização do processo de ozonização, empregou-se o equipamento Degradatox ${ }^{\oplus}$ (OZ Engenharia, Porto Alegre, RS, Brasil). Este equipamento é composto por um gerador de ozônio, um reator tipo reservatório com suporte, bomba e conexôes hidráulicas e uma bomba submersa. A bomba submersa força a entrada do efluente a partir do tanque de decantação para dentro do reator, onde o efluente permanece em recirculação e em contato com o ozônio. Passado o período de tempo desejado, o líquido tratado é escoado para o leito de volatilização. A amostragem foi composta por 7 amostras obedecendo a ordem descrita na Tabela 1 e em conformidade com os pontos de coleta da Figura 1. 
Tabela 1. Caracterização das amostras do efluente em cada ponto de amostragem e seus momentos de ozonização e tempo de retenção

\begin{tabular}{|c|c|c|c|}
\hline $\begin{array}{l}\text { Amostra/ } \\
\text { Pontos }\end{array}$ & $\begin{array}{l}\text { Característica } \\
\text { dos efluentes }\end{array}$ & $\begin{array}{l}\text { Tempo de } \\
\text { ozonização }\end{array}$ & $\begin{array}{l}\text { Tempo de } \\
\text { residência }\end{array}$ \\
\hline 0 & Resíduo bruto $^{2}$ & --- & $\begin{array}{l}\text { Coleta ime- } \\
\text { diata }\end{array}$ \\
\hline 1 & $\begin{array}{l}\text { Efluente após } \\
\text { adição de água } \\
\text { ao resíduo bruto }^{3}\end{array}$ & --- & $\begin{array}{l}\text { Coleta ime- } \\
\text { diata }\end{array}$ \\
\hline 2 & $\begin{array}{l}\text { Efluentes após } \\
\text { deposição no } \\
\text { tanque de decan- } \\
\text { tação }^{4}\end{array}$ & --- & 72 horas \\
\hline 3 & $\begin{array}{l}\text { Efluente em } \\
\text { ozonização }\end{array}$ & 1 hora & 1 hora \\
\hline 4 & $\begin{array}{l}\text { Efluente em } \\
\text { ozonizaçáa }\end{array}$ & 3 horas & 3 horas \\
\hline 5 & $\begin{array}{l}\text { Efluente em } \\
\text { finalização da } \\
\text { ozonização }\end{array}$ & 6 horas & 6 horas \\
\hline 6 & $\begin{array}{l}\text { Efluentes após } \\
\text { deposição no } \\
\text { leito de volatil- } \\
\text { ização }^{6}\end{array}$ & --- & 72 horas \\
\hline
\end{tabular}

NOTA: ${ }^{1}$ tempo de residência da amostra em cada componente do sistema até a sua coleta para análise; ${ }^{2}$ resíduo bruto, calda pulverizada do inseticida Cascade ${ }^{\circledR} 100$, preparada de acordo com as orientações descritas na bula do produto; ${ }^{3}$ mistura do resto do produto da aplicação mais a quantidade média de água empregada na lavagem da aeronave; ${ }^{4}$ efluente após passagem pelo tanque de decantação e antes de iniciar o processo de oxidação (ozônio); ${ }^{5}$ efluente em oxidação; ${ }^{6}$ efluente após exposição no leito de volatilização.

A concentração do princípio ativo na calda obedeceu à taxa modal de aplicação de $10 \mathrm{~L} / \mathrm{ha}$, que é o volume de calda de inseticida por hectare mais frequentemente pulverizado nas operações realizadas nas empresas amostradas nos municípios de Rosário do Sul (RS) e Dom Pedrito (RS). O efluente foi tratado em bateladas de volume igual a 400 L. Esta quantidade representou o volume de efluente produzido por três lavagens completas de aeronaves. Este dado é a média de efluente gerado em cada lavagem $(132,8 \mathrm{~L})$ multiplicado por três $(398,4 \mathrm{~L} \approx 400 \mathrm{~L}$ ) (FURTADO,
2012).

-Determinação do teor de flufenoxurom

A determinação analítica do princípio ativo flufenoxurom foi realizada através do uso de cromatografia líquida de alta eficiência acoplada à espectrometria de massas (LC-MS/MS). Uma vez que a concentração das amostras estava na faixa de $\mathrm{mg} / \mathrm{L}$ não foi necessária a aplicação de técnicas de concentração das amostras. As análises foram realizadas utilizando um sistema de LC-MS/MS Varian 320 (Walnut Creek, EUA), equipado com bomba binária 212 LC, amostrador automático ProStar 410 e espectrômetro de massas triplo quadrupolo, operando com fonte de ionização por electrospray. A aquisição de dados foi realizada com o emprego do software Varian Workstation 6.6. A separação foi efetuada em coluna Pursuit XRs Ultra C18 $(100 \times 2 \mathrm{~mm} ; 2,8 \mu \mathrm{m}$ de tamanho de partícula) da Agilent (Santa Clara, EUA). A coluna foi mantida a $30^{\circ} \mathrm{C}$ e o volume de injeção foi de $5 \mu \mathrm{L}$.

\section{-Análise dos dados}

A análise descritiva foi utilizada com emprego de gráficos e tabelas para avaliação do comportamento das diferentes variáveis medidas. Para a interpretação dos dados do decaimento dos princípios ativos presentes nos efluentes dos agrotóxicos, empregou-se equações de regressão, úteis para analisar relações entre variáveis contínuas, típicas deste estudo. Para geração dos gráficos, das tabelas e do cálculo da equação da regressão, empregaram-se as ferramentas estatísticas do software Excel $2013^{\circledR}$.

\section{RESULTADOS E DISCUSSÃO}

A Tabela 2 apresenta a concentração do princípio ativo flufenoxurom presente na calda original (C.O.) e nos 7 pontos amostrados do sistema de descontaminação de aeronaves (Tabela 1). Percebese diminuição na concentração de flufenoxurom equivalente a $82,2 \%$ no tratamento com $1,0 \mathrm{~g} \mathrm{O}_{3} / \mathrm{h} \mathrm{e}$ de $86,0 \%$ no tratamento com $2,0 \mathrm{~g} \mathrm{O}_{3} /$ hora, quando comparadas às concentraçóes na calda original (C.O.) e no leito de volatilização (amostra 6). 
Tabela 2. Concentrações de flufenoxurom (em mg/L) para os diferentes pontos de amostragem do sistema de descontaminação de aeronaves. Tratamentos oxidativos com 1,0 e $2,0 \mathrm{~g} \mathrm{O}_{3} / \mathrm{h}$.

Flufenoxurom $(\mathrm{mg} / \mathrm{L})$

\begin{tabular}{llllll}
\hline Pontos & $\begin{array}{l}{\left[\mathrm{O}_{3}\right]=1,0} \\
\left(\mathrm{~g} \mathrm{O}_{3} / \mathrm{h}\right)\end{array}$ & $\begin{array}{c}\text { \% Var } \\
\text { Total }\end{array}$ & $\begin{array}{l}{\left[\mathrm{O}_{3}\right]=2,0} \\
\left(\mathrm{~g} \mathrm{O}_{3} / \mathrm{h}\right)\end{array}$ & $\begin{array}{l}\% \mathrm{Var} \\
\text { Total }\end{array}$ & $\begin{array}{l}\text { \% Var. } \\
\mathrm{e} \mathrm{2}\end{array}$ \\
\hline C.O. & 5080,0 & - & 5080,0 & - & 0,0 \\
1 & 836,7 & $-83,5$ & 1019,3 & $-79,9$ & 17,9 \\
2 & 610,1 & $-88,0$ & 610,1 & $-88,0$ & 0,0 \\
3 & 919,3 & $-81,9$ & 818,9 & $-83,9$ & $-12,3$ \\
4 & 781,7 & $-84,6$ & 876,1 & $-82,8$ & 10,8 \\
5 & 792,1 & $-84,4$ & 764,3 & $-85,0$ & $-3,6$ \\
6 & 874,7 & $-82,8$ & 710,3 & $-86,0$ & $-23,1$ \\
\hline
\end{tabular}

Obs: $\left[\mathrm{O}_{3}\right]=1,0$ : média das amostras no tratamento com $1,0 \mathrm{~g} \mathrm{O}_{3} / \mathrm{h}$; $\left[\mathrm{O}_{3}\right]=2,0$ : média das amostras no tratamento com $2,0 \mathrm{~g} \mathrm{O}_{3} / \mathrm{h} ; \%$ Var Total = variação entre as unidades amostrais e C.0.; \% Var. 1 e 2 = variação entre as Médias 1,0 e Média 2,0.

Entretanto, uma vez que se desconte o efeito da diluição que ocorre após a adição da água da lavagem, verifica-se que o flufenoxuron mostra-se resistente à ação do ozônio. No tratamento com 2,0 g de $\mathrm{O}_{3} /$ hora, foi verificado uma ação mais pronunciada da ozonólise. O resultado final da concentração de flufenoxuron presente no efluente na amostra 6, para o tratamento com $1,0 \mathrm{~g}$ de $\mathrm{O}_{3} /$ hora, é igual a 874,7 $\mathrm{mg} / \mathrm{L}$ de ingrediente ativo e no tratamento com 2,0 $\mathrm{g}$ de $\mathrm{O}_{3} /$ hora é igual a $710,3 \mathrm{mg} / \mathrm{L}$ de ingrediente ativo. Essa diferença $(23,1 \%)$ indica que o tratamento com carga maior de ozônio poderá gerar diferença final no resultado, embora as condiçôes experimentais devam ser melhor otimizadas para promover efeitos de degradação substanciais sobre a molécula em estudo.

A Figura 2 mostra a similaridade nas tendências de decaimento do flufenoxurom nos dois tratamentos empregados. Pode-se se observar que há uma flutuação nas concentraçóes do princípio ativo nas amostras, sendo que aparentemente a menor concentração é verificada no tanque de decantação. Entretanto, as flutuaçóes das leituras entre as amostras 3, 4, 5 e 6 podem ser atribuídas ao erro experimental ou à incerteza da mediçáo, uma vez que o tanque de decantação per se não produz nenhuma ação física ou química sobre a molécula do agrotóxico em estudo.

Figura 2. Concentração de flufenoxuron no efluente (mg/L) nos pontos de amostragem no sistema de descontaminação de aeronaves. Condições experimentais: 1,0 e $2,0 \mathrm{~g}$ de $0_{3} /$ hora, temperatura ambiente, tempos de ozonização de 1, 3 e 6 horas.

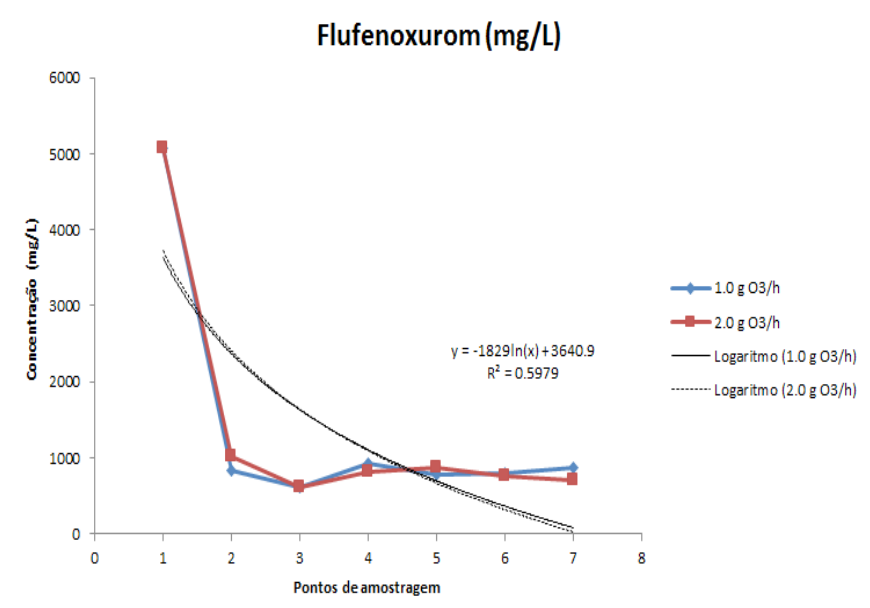

Durante os processos de tratamento foi verificado um decaimento da carga total do princípio ativo flufenoxurom igual a $44,8 \%$ (para $1,0 \mathrm{~g} \mathrm{O}_{3} / \mathrm{h}$ ) e de $63,2 \% \quad\left(2,0 \mathrm{~g} \mathrm{O}_{3} / \mathrm{h}\right)$. O tratamento com 2,0 $\mathrm{g} \mathrm{O}_{3} / \mathrm{h}$ mostrou-se, portanto, $23,1 \%$ mais eficiente que o tratamento com $1,0 \mathrm{~g} \mathrm{O}_{3} / \mathrm{h}$.

A amostra 6 apresenta uma variação negativa igual a $37 \%$ e $47 \%$ para tratamentos com 1,0 e $2,0 \mathrm{~g} \mathrm{O}_{3} / \mathrm{h}$, respectivamente, em relação à amostra 5. Dentro do leito de volatilização, o princípio ativo mostrou decaimento, evidenciando a funçáo de polimento desta unidade de tratamento (Tabela 3).

Estes dados mostram que o tratamento com 2,0 g foi mais vantajoso em termos de eficiência, além de demonstrar a importância do leito de volatilizaçáo no trabalho de polimento da remoçáo do princípio ativo. 
Tabela 3. Carga de flufenoxurom por tratamento (batelada) no efluente (em gramas de princípio ativo) nos diversos pontos de amostragem no sistema de descontaminação de aeronaves. Tratamentos oxidativos com 1,0 e $2,0 \mathrm{~g} \mathrm{O}_{3} / \mathrm{h}$

Carga flufenoxurom (gramas de principio ativo)

\begin{tabular}{|c|c|c|c|c|c|c|c|}
\hline Pontos & $\begin{array}{l}{\left[\mathrm{O}_{3}\right]} \\
=1,0 \\
(\mathrm{~g} \\
\left.\mathrm{O}_{3} / \mathrm{h}\right)\end{array}$ & $\% \operatorname{Var}$ & $\begin{array}{l}\% \text { Var } \\
\text { Total }\end{array}$ & $\begin{array}{l}{\left[\mathrm{O}_{3}\right]} \\
=2,0 \\
(\mathrm{~g} \\
\left.\mathrm{O}_{3} / \mathrm{h}\right)\end{array}$ & $\%$ Var & $\begin{array}{l}\% \text { Var } \\
\text { Total }\end{array}$ & $\begin{array}{l}\% \\
\text { Var.1 } \\
\text { e } 2\end{array}$ \\
\hline 1 & 334,7 & 0,0 & 0,0 & 407,7 & 0,0 & 0,0 & 17,9 \\
\hline 2 & 234,3 & $-30,0$ & $-30,0$ & 234,3 & $-42,5$ & $-42,5$ & 0,0 \\
\hline 3 & 353,0 & 50,7 & 5,5 & 314,4 & 34,2 & $-22,9$ & $-12,3$ \\
\hline 4 & 300,2 & $-15,0$ & $-10,3$ & 336,4 & 7,0 & $-17,5$ & 10,8 \\
\hline 5 & 293,1 & $-2,4$ & $-12,4$ & 282,8 & $-15,9$ & $-30,6$ & $-3,6$ \\
\hline 6 & 184,7 & $-37,0$ & $-44,8$ & 150,0 & $-47,0$ & $-63,2$ & $-23,1$ \\
\hline
\end{tabular}

Obs: $\left[\mathrm{O}_{3}\right]=1,0$ : média das amostras no tratamento $\operatorname{com~} 1,0 \mathrm{~g} \mathrm{O}_{3} / \mathrm{h}$; $\left[\mathrm{O}_{3}\right]=2,0$ : média das amostras no tratamento com 2,0 $\mathrm{g} \mathrm{O}_{3} / \mathrm{h} ; \% \mathrm{Var}$ = variação entre as unidades amostrais (intra); \% Var Total = variação entre as unidades amostrais (inter); \% Var. 1 e 2 = variação entre as Médias 1 e Média 2.

A oxidação do princípio ativo flufenoxurom em ambas as taxas de aplicação com $1,0 \mathrm{~g}$ e com 2,0 g de $\mathrm{O}_{3} /$ hora apresentaram resultados medianamente satisfatórios, visíveis na Figura 3, durante a passagem do efluente pelo sistema de ozonização, porém com a deposição no leito de volatilização (amostra 6) ocorre uma melhora na remoção da carga do princípio ativo na batelada tratada.

Figura 3. Carga de flufenoxurom no efluente do inseticida Cascade ${ }^{\circledR} 100$ (em gramas de princípio ativo/ bateladal nos pontos de amostragem no sistema de descontaminação de aeronaves. Tratamentos oxidativos com 1,0 e $2,0 \mathrm{~g} \mathrm{O}_{3} / \mathrm{h}$

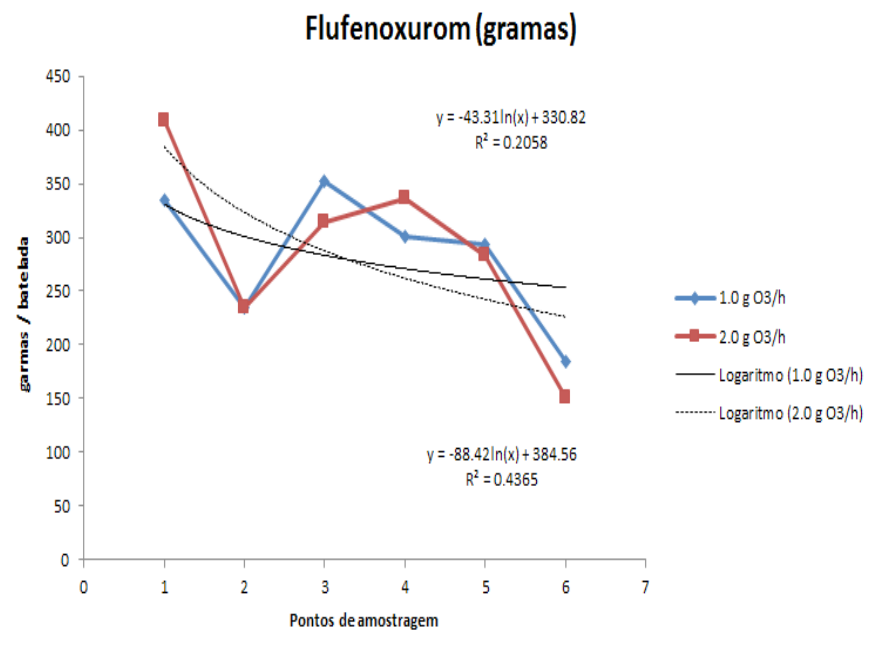

Mesmo que o flufenoxuron apresente certa resistência à oxidação, em ambas as equaçóes temos $\alpha<0$, demonstrando a tendência de queda da carga total do princípio ativo flufenoxuron no efluente.

Em relação ao aparente aumento das cargas de flufenoxurom que foi verificado nas amostras coletadas dentro do reator de ozonização (amostras 3, 4 e 5) quando comparadas com as amostras do tanque de decantação (amostra 2), uma das possibilidades é o erro experimental, especialmente o vinculado à incerteza da medição do método analítico. Entretanto, há que se considerar a possibilidade de que nas 72 horas em que o efluente gerado pela lavagem fica em decantaçáo (antes da entrada no reator de ozonizaçáo) possa ocorrer a formação de produtos de transformação do flufenoxurom (hidrólise) e que tais produtos possam reverter à forma original da molécula quando expostos à ação do ozônio.

Durante o período de realização dos experimentos, a temperatura ambiente variou entre 18 e $32^{\circ} \mathrm{C}$, a umidade relativa do ar variou de 44 a $72 \%$ e o vento apresentou uma velocidade media de $3 \mathrm{~km} / \mathrm{h}$. O período de luz solar direta foi estimado em cerca de 12 horas, com baixíssima presença de nuvens, fato importante para compreendermos a intensa evaporação verificada no leito de volatilização em um período de 72 horas.

Quanto ao $\mathrm{pH}$ das amostras, o valor inicial do $\mathrm{pH}$ na calda original foi de 4,0. Após a adição da água de lavagem e passagem pelo piso de escorrimento, o valor subiu para 6,0. Após a o processo de ozonização, as amostras sofreram uma acentuada acidificação, sendo que o $\mathrm{pH}$ das amostras ao saírem do sistema 
de ozonização apresentaram valor de $\mathrm{pH}$ de 3,5. Não houve diferença significativa nas alteraçóes de $\mathrm{pH}$ quando comparados os dois níveis de concentraçáo de ozônio avaliados.

\section{CONCLUSÃO}

A ozonização com taxa de $1,0 \mathrm{~g} \mathrm{O}_{3} / \mathrm{h}$ mostrouse eficiente na degradação do princípio ativo flufenoxuron presente no inseticida Cascade ${ }^{\circledR} 100$, reduzindo concentração e carga do princípio ativo nos efluentes obtidos após a lavagem interna e externa das aeronaves. Entretanto, verificamos que a ozonizaçáo com nível de 2,0 $\mathrm{g} \mathrm{O}_{3} / \mathrm{h}$ mostra-se mais eficiente na degradaçáo do flufenoxurom quando comparado com o tratamento empregando $1,0 \mathrm{~g} \mathrm{O}_{3} / \mathrm{h}$. Não obstante, o segundo tratamento náo foi proporcionalmente superior ao primeiro.

O leito de volatilização mostrou-se eficiente na retenção do efluente, não permitindo seu contato com o solo e permitindo a exposição às condições atmosféricas (radiação ultravioleta e oxigênio atmosférico), promovendo maior grau de degradação do agrotóxico, levando a uma diminuição da concentração e da carga do princípio ativo.

Conclui-se que o sistema proposto na Instrução Normativa no 02 de janeiro de 2008 mostrou-se funcional e eficiente para a degradação do princípio ativo do inseticida Cascade ${ }^{\circledast} 100$, atendendo as legislaçóes ambientais e agrícolas a nível federal e do estado do Rio Grande do Sul. O uso da ozonização aplicada à efluentes é uma maneira viável e eficaz de provocar degradação de agrotóxicos, diminuindo de maneira significativa o risco do descarte de tais efluentes no meio ambiente.

Foi também demonstrado que o flufenoxurom é parcialmente resistente à ação do ozônio e que novos estudos devem ser realizados de modo a otimizar o sistema de tratamento proposto para a obtenção de uma degradação mais extensiva deste agrotóxico. Do mesmo modo, é de grande importância a realização de estudos sobre os possíveis produtos de transformação do flufenoxurom e quais os mecanismos moleculares implicados na formação destes.

\section{LITERATURA CITADA}

ANTUNIASSI, U. R. Evolution of agricultural aviation in Brazil. Outlooks on Pest Management, 2015. v. 26, n. 1, p. 12-15.

BARRANCO, M. et al. Simple and rapid determination of benzoylphenylurea pesticides in river water and vegetables by LC-ESI-MS. Chromatographia, 2007. v. 66, n. 7-8, p. 533-538. BUENO, M. R.; CUNHA, J. P. A. R. Da; SANTANA, D. G. DE. Assessment of spray drift from pesticide applications in soybean crops. Biosystems Engineering, Fevereiro. 2017. Engineering Approaches for Reducing Spray Drift. v. 154, p. 35-45.

CARMO, E. L.; BUENO, A. F.; BUENO, R. C. O. F. Pesticide selectivity for the insect egg parasitoid Telenomus remus. BioControl, 2010. v. 55, n. 4, p. 455-464.

COSTA, E. M. et al. Toxicity of insecticides used in the Brazilian melon crop to the honey bee Apis mellifera under laboratory conditions. Apidologie, 2014. v. 45, n. 1, p. 34-44.

FURTADO, R. D. Tratamento de efluentes gerados pela lavagem de aeronaves agrícolas e pelo descarte das aplicaçôes aéreas de agrotóxicos. Porto Alegre: Universidade Federal do Rio Grande do Sul, 2012. Tese.

GIL, G. et al. Determination of benzoylureas in ground water samples by fully automated on-line pre-concentration and liquid chromatography-fluorescence detection. Journal of Chromatography A, 2006. v. 1103, n. 2, p. 271-277.

Inseticida Cascade 100: BASF. [S.l.], [s.d.]. Disponível em: <http://www.agro.basf.com.br/agr/ms/apbrazil/pt/content/ APBrazil/solutions/insecticides/insecticides_product/ CASCADE_100>. Acesso em: 6 out. 2017.

JEONG, H. H. et al. Rapidly progressive lactic acidosis in patients with flufenoxuron poisoning. Hong Kong Journal of Emergency Medicine, 2014. v. 21, n. 3, p. 181-184.

KAMEL, A. et al. Degradation of the acaricides abamectin, flufenoxuron and amitraz on Saudi Arabian dates. Food Chemistry, 2007. v. 100, n. 4, p. 1590-1593.

MAILLARD, E. et al. Removal of pesticide mixtures in a stormwater wetland collecting runoff from a vineyard catchment. Science of the Total Environment, 2011. v. 409, n. 11, p. 23172324.

MINISTÉRIO DA AGRICULTURA, PECUÁRIA E ABASTECIMENTO. INSTRUÇÃO NORMATIVA No 02/2008.

OLLER, I.; MALATO, S.; SÁNCHEZ-PÉREZ, J. A. Combination of Advanced Oxidation Processes and biological treatments for wastewater decontamination-A review. Science of the Total Environment, 2011. v. 409, n. 20, p. 4141-4166.

RICE, N. et al. Unplanned releases and injuries associated with aerial application of chemicals, 1995-2002. Journal of Environmental Health, 2005. v. 68, n. 4, p. 14-18.

STATHIS, I. et al. Novel imazethapyr detoxification applying advanced oxidation processes. Journal of Environmental Science and Health, Part B, Agosto. 2011. v. 46, n. 6, p. 449-453.

WOO, J.-H.; LIM, Y. S. Severe human poisoning with a flufenoxuron-containing insecticide: Report of a case with transient myocardial dysfunction and review of the literature. Clinical Toxicology, 2015. v. 53, n. 6, p. 569-572. 